\title{
Establishment and Analysis of 3D Structure in Co-Parenting Behavior of Pupil's Parents
}

\author{
Ping Zou1,2, Lizhu Yang3*, Wen Liu3, Wei Zou ${ }^{3,4}$, Xinlei Li ${ }^{2}$ \\ ${ }^{1}$ Teachers College, Dalian University, Dalian, China \\ ${ }^{2}$ Higher Education Research Institute, Dalian University, Dalian, China \\ ${ }^{3}$ The Research Center of Developmental and Educational Psychology, Liaoning Normal University, Dalian, China \\ ${ }^{4}$ College of Life Science, Liaoning Normal University, Dalian, China \\ Email: ${ }^{*}$ pingzoudldx@163.com
}

Received 31 October 2013; revised 30 November 2013; accepted 8 December 2013

Copyright (C) 2014 by authors and Scientific Research Publishing Inc.

This work is licensed under the Creative Commons Attribution International License (CC BY).

http://creativecommons.org/licenses/by/4.0/

(c) (1) Open Access

\section{Abstract}

Questionnaire method was used to study co-parenting structure with a sample $\mathbf{7 7 5}$ of couples of school-age children in Dalian of China. At the same time, through situational observations, coding analyses and evaluating for 28 nuclear families' parents, co-parenting behavior's actuality and characteristics were shown. The results show that (1) Three factors covered in co-parenting structure: Harmonious-support, active-involvement and opposite-conflict; (2) Superior reliability and validity in self-designed, parental reported co-parenting questionnaire, a reliable test instrument measuring and analyzing the co-parenting behavior, three factors related model, turn to be reasonable and valid.

\section{Keywords}

Pupil; Co-Parenting Behavior; Structure

\section{Introduction}

In 1985, Weissman and Cohen firstly raised the concept of Parenting Alliance (Abeer \& Jerome, 2003). In 2000, McHale developed the theory that co-parenting is the coordinating quality of parents' parenting role (McHale, Kuersten-Jogan, Lauretti, \& Rasmussen, 2000). As a new field of family and children development, co-parenting only has a history of two or three decades abroad.

In China, the study in this field has just began, which is receiving the attention from educational and developmental psychology. So far, the structure, measuring and development of co-parenting have been largely

${ }^{*}$ Corresponding author. 
unknown. In the structure matter of co-parenting, scholars domestic and overseas raised multidimensional structure models, including the models from two-dimension to five-dimension. However, in the study of object matter, scholars overseas mainly focus on the study of co-parenting of children, e.g., many children (Lindsey, Gale, \& Cowell, 2005; McHale, 2007; Karreman, Tuijl, \& Marcel, 2008; Feinberg \& Kan, 2008). They usually take the preprimary children's family as study objects and observe the parent-child tripartite interaction of infant families (Schoppe-Sullivan, Manglsdorf, Brown, \& Sokolowski, 2007; Kolaka \& Vernon-Feagans, 2008). And only a few school children's parents are taken as study objects (Russell \& Russell, 1994; Schoppe-Sullivan, Weldon, Cook, Davis, \& Buckley, 2009; Margolin, Gordis, \& John, 2001).

At present, there are less studies about the current situation of the children co-parenting behavior, and only a few Taiwanese scholars (Cuixia Lv, 2002) who have a small number of study samples are probing into the co-parenting behavior of preprimary children. In Chinese Mainland there are still no empirical studies systematically probing into the structure of co-parenting of primary school children. To study the structure of co-parenting factors, on one hand, it helps to probe into the essence of co-parenting and provides logical proofs for compiling assessment questionnaire; on the other hand, it provides specific direction and objective for the parenting which is beneficial to children's personality development.

Combining the past study on co-parenting, we define the co-parenting behavior as a mutually supportive, cooperative and coordinating psychological quality in the tripartite interaction process among parents and children, as well as the parents' specific parenting behaviors. As a matter of fact, co-parenting emphasizes more on the interaction of parents and children from the subsystem of the family structure.

Western scholars raised different theoretical models about the study on the behavior structure of co-parenting. Through confirmatory factor analysis on the test result of the Parenting Alliance (PAM), Konold (2001) et al. found that the fathers' and the mothers' parenting alliances are both comprised of two same dimensional factors: one is parents' communication and family team spirit, the other one is emotional respect (Konold \& Abidin, 2001). Melanie, McConnell and Patricia believe that co-parenting includes not only the effect of the adults' mutual support, e.g., enthusiasm and cooperation, but also the effect without mutual support, e.g., the inconsistent parenting viewpoints or destructive forces in the mutual communication (McConnell \& Kerig, 2002). Brody et al. construct co-parenting from three aspects: parents' communication and instrumental support, conflicts between the father's and the mother's parenting, quality of marriage interaction (Brody, Stoneman, Flor, McCrary, Hastings, \& Conyers, 1994). Van Egeren believes that co-parenting behavior has four dimensions: the unity of co-parenting, the support of co-parenting, the damage to co-parenting, and the sharing responsibility for parenting (Van Egeren \& Hawkins, 2004). McHale raised the four aspects of co-parenting behavior: family cohesion, belittlement, conflict and consolidation. Behavior observation reflects five variables: opposition, warmth and cooperation, child/adult center, balance of positive parenting participation, and dealing with children's behavior (McHale, 1997).

From the studies on co-parenting behavior domestic and overseas, we can discover that no matter the theoretical constructions of the former scholars are two-dimensional, three-dimensional, four-dimensional or five-dimensional, they have something in common: in content they include the couple's support and cooperation in the co-parenting (the Parenting Alliance contains cooperative behaviors such as respect, cooperation, emphasis, promotion, affirmation, supplement, repetition of each other and division of work, and unity.); some affect management modes of the tripartite interactive relationship of the parents and the children reflect the parents' participant positivity on the attempt aspect in the family team construction, and others reflect the undesirable interaction of the parents and the child, e.g., opposition of the parenthood (father and child alliance or mother and child alliance); and the obvious external divergence and conflict of the couple (the rivalry of behavior and quarrel, etc.). These problems mainly focus on the tripartite interaction about parenting with the child present.

\section{Method}

\subsection{Establishment of the Structure Model}

\subsubsection{Materialization of Information-Open-Ended Questionnaire and Conclusion of Parents Interviewing Material}

In order to sufficiently understand the behavior characteristics of co-parenting, we made a survey with openended questionnaire; meanwhile, we interviewed some primary school students' parents personally. We selected the parents of primary school students from grade 1 to grade 6 in two ordinary primary schools in Dalian City, 
gave out 150 copies of questionnaires and regained 100 copies of effective questionnaires, whose effective rate is $67 \%$. We did semi-structural interview with 27 parents of the students in six primary schools in the Development Zone of Dalian City.

We coded the material on the basis of summarizing the open-ended questionnaire and the interviewing material. Firstly, we abstracted the keywords generally and tried to systemize them: we sorted the expressions describing the similar contents into one category, in which processes, we deleted the repetitive and invalid vocabulary and finally formed the cognition of four aspects on theoretical construction, basing on the former study and the open-ended questionnaires. Secondly, using these four aspects as a structure, we did semi-structural interview with 27 parents of primary school students and let them talk about their own views relating to the parenting reality. The interview is mainly aimed at these questions, such as whether the couples are unitive and cooperative on the aspect of co-parenting, the management mode of parent-child interaction, the positivity of participation, whether there is opposition or conflict, and so on. Lastly, we systemized and literally transcribed the interview material, and used Nvivo1.2 for the statistical coding of the text file of content in computer.

\subsubsection{Theory Searching-Absorbing the Predecessors' Research Results}

On the basis of reading a lot of literature material, we analyzed the existing co-parenting behavior structure. In the study of co-parenting structure, many research results provided analysis of co-parenting from different angles and sides. First, we preliminarily summarized and listed the relative schedules of the existing co-parenting structure dimension. And then, we compared the structures with similar contents and restricted the meaning of co-parenting behavior. When we found the co-parenting behavior mode, we sufficiently referred to these research results, combined the characteristics of family parenting of primary school students, absorbing rational contents and rejecting irrational ones, and formed a preliminary theory cognition.

\subsubsection{Exploratory Factor Analysis}

Referring to the theoretical results of the previous studies, applying open-ended questionnaire and interview for coding, we reached the four structural dimensions of the co-parenting behavior questionnaire, on the basis of theory research.

The first dimension is cooperation and support, i.e., the degree of the parents' mutual endeavor of cooperation and support in the tripartite interaction of parenting.

The second dimension is active participation, i.e., the parents' behavioral and participant degree on the aspect of exerting the effect of family team, which mainly means the parent's participant initiative and positivity in the parent-child activities.

The third dimension is parenting opposition, i.e., triangle, which means the inappropriate alliance relationship forming in parenting. The mainly forms are father-child alliance or mother-child alliance.

The fourth dimension is parenting conflict, i.e., there are direct conflicts in the couple's behaviors because of their inconsistent views on parenting questions, or indirect parent-child conflicts caused by parenting.

According to the analysis on open-ended questionnaires, interview coding and theory searching and pre-test, we put forward that the co-parenting behavior of primary school students included four dimensional theory constructions. Each dimension is not independent, but has organic connection and interaction effect with each other. Finally, we selected and compiled 35 questions to form the preliminary questionnaire. The number of questions in each dimension is 12, 7, 6 and 10, respectively.

\subsection{Formation of Questionnaire}

\subsubsection{Collection and Compilation of Test Questions}

According to the operational definition and theoretical constructions of co-parenting, we selected representative and universal co-parenting behaviors to design the test clause, and made a preliminary co-parenting behavior questionnaire of 45 questions. On this basis, we asked experts and some parents for their opinions, invited experts on developmental psychology and postgraduates majoring in psychology, primary school teachers and primary school students' parents to check and approve the preliminarily revised questionnaire from the angles of item content and appropriateness of expression, based on which, we further modified the questionnaire. We checked and evaluated the contents and sentences of the questionnaire one by one, deleting 10 unclear or repeating items and modifying certain questions without changing the item content, then we formed a pre-test 
questionnaire of 35 questions.

\subsubsection{Pre-Test}

From 2 primary schools in Dalian City, we selected the primary school students' parents in nuclear families, gave out 600 copies of questionnaires (300 nuclear families), and regained 400 copies of them (200 nuclear families).

On the basis of open-ended questionnaires and revised interview items, we revised the item content and its expression according to results of the pre-test. We filtrated the items through preliminary test and confirmed the closed-end questionnaire in due form, adopting exploratory factor analysis. We preliminarily finished the validity test and reliability test of the pre-test result. The coefficient of internal consistency of the pre-test questionnaire is 0.89 , and its Guttman split-half coefficient is 0.85 ; the correlation among all dimensions and the correlation between the dimensions and the total points is between $0.4-0.83$. We analyzed the correlation of the questionnaire and deleted the questions with exorbitant relevancy; we did frequency test, deleted the questions far from the normal distribution or with low discrimination degree, and then formed the testing questionnaire in due form including 22 questions in three dimensions.

\subsection{Testing}

\subsubsection{Selection of Testees}

In the formal testing, we totally selected 2000 parents of 1000 primary school students in nuclear families in 9 primary schools in Dalian City and Benxi City for the questionnaire survey. We regained 1644 copies of effective questionnaires (822 copies from the fathers and 822 copies from the mothers). Here, the number of parents tested for exploratory factor analysis is 828 (414 fathers and 414 mothers), and the number of parents tested for confirmatory factor analysis is 816 (408 fathers and 408 mothers).

\subsubsection{Testing Instrument}

In this study, we adopted self-compiled questionnaire to investigate the question of co-parenting behavior of primary school students. The co-parenting quality questionnaire (self-compiled) includes 35 questions in four dimensions. There are mainly five-level score standard in the questionnaire. The answer of forward questions consists of "never do like this", "rarely do like this", "sometimes do like this", "often do like this" and "always do like this", whose scores are successively assigned as 1, 2, 3, 4 and 5. The scoring sequence of the backward questions is the opposite.

\subsubsection{Testing Procedure}

The data of exploratory factor analysis in the formal testing were collected from five primary schools in Dalian City. Through analysis we determined 20 questions to form the questionnaire evaluating the co-parenting behavior of primary school students' parents finally.

The data of confirmatory factor analysis in the formal testing were collected from another three primary schools in Dalian City and one primary school in Benxi City. With the help of these data, we finished the structure verification of two samples for the questionnaire of three dimensions and 20 questions.

After the formal testing, we selected $20 \%$ of the tested parents to answer the questionnaire two weeks later.

\subsubsection{Data Processing}

In this study, we used software SPSS10.0 to analyze the basic information of the data and process the discussion of questionnaire dimension. Using software LISREL8.70, we processed the discussion of structure with covariance structure model.

\section{Results}

\subsection{Exploratory Factor Analysis}

Firstly, for the appropriateness investigation of the factor analysis of the data, the value of KMO is 0.901 , which indicated that the factor analysis is able to explain the relationship among the variables sufficiently; the value of Bartlett Test Sphericity is $5107.309(p<0.000)$, which is on the significance level and indicates that correlation 
matrix of the variables is conspicuous and we can conduct factor analysis on the data of this study. Results see Table 1.

Secondly, we determine the number of factors according to the following standards: first, the eigenvalue of the factor is greater than 1 ; second, the factor solution conforms to sharp step test, and the factors are determined according to the display of scree plot; third, each factor includes at least 3 questions. The analysis on the principal component of these 20 questions shows that the eigenvalue of 4 factors is greater than 1 , which totally explains $54.195 \%$ of the total square deviation. Thereinto, the eigenvalue of the first factor is 5.955, and the explained variance (i.e., contribution rate) is $29.997 \%$; the eigenvalue of the second factor is 2.520 , and the explained variance is $41.770 \%$; the eigenvalue of the third factor is 1.448 , and the explained variance is $49.067 \%$; the eigenvalue of the forth factor is 1.018, and the explained variance is $54.195 \%$.

Based on the "simplest structure" principle, three factors can be extracted. In order to further verify whether the three factors extracted are the best structures, we respectively selected 2 and 4 factors for orthogonal rotation. The results showed the phenomenon of quite imbalanced item allocation of each factor, or the internal meaning of some factors was difficult to be explained reasonably. It also proved the rationality of the three factors adopted in this study from the other aspect. Table 1 showed that there is a group of higher loading variables under each factor, and the common information of the variables forms the connotative character of the factor.

Therefore, we renamed these factors respectively: cooperation and support, active participation, opposition and conflict, which include 5, 6 and 9 questions respectively. In this way, we reached the co-parenting behavior structure for the primary school students.

We compared the theoretically conceived co-parenting dimension and the results of the factor analysis and observed degree of coincidence, whose results see Table 2.

Table 2 shows that after factor rotation, the 5 questions (4, 5, 9, 10 and 13) of cooperation and support and the 6 questions (1, 2, 3, 12, 19 and 20) didn't change. While, after factor rotation, the 4 questions $(6,11,15$ and 18 ) forming the opposite relation of factor 2 , i.e., opposition and conflict, form factor 3 , i.e., opposition and conflict, along with the former divergent and conflicting 5 questions $(7,8,14,16$ and 17).

Overall, the 3 factors extracted are basically identical with the theoretical conception. The above classification is just based on the meaning of statistics. Whether this theoretical model or assumption is rational and effective, we still need to verify and investigate it through confirmatory factor analysis.

Table 1. Factor loading of the co-parenting behavior questionnaire.

\begin{tabular}{cccccc}
\hline Item & Factor 1 & Item & Factor 2 & Item & Factor 3 \\
\hline 4 & 0.742 & 11 & 0.761 & 26 & 0.705 \\
5 & 0.711 & 9 & 0.756 & 28 & 0.684 \\
9 & 0.673 & 10 & 0.704 & 17 & 0.671 \\
10 & 0.520 & 33 & 0.643 & 16 & 0.653 \\
13 & 0.486 & 34 & 0.644 & 25 & 0.651 \\
& & 22 & 0.517 & 15 & 0.625 \\
& & & 29 & 0.611 \\
& & & 20 & 0.598 \\
& & & 31 & 0.562 \\
\hline
\end{tabular}

Table 2. The correspondence the 4 dimensions and 3 factors of the theoretical conception.

\begin{tabular}{cccccccccc}
\hline \multicolumn{1}{c}{ Quctor } & \multicolumn{10}{c}{ Question Number } \\
\hline Cooperation \& Support & $4^{2}$ & $5^{1}$ & $9^{1}$ & $10^{1}$ & $13^{1}$ \\
Active Participation & $1^{2}$ & $2^{2}$ & $3^{2}$ & $12^{2}$ & $19^{2}$ & $20^{2}$ & \\
Opposition \& Conflict & $6^{3}$ & $11^{3}$ & $15^{3}$ & $18^{3}$ & $7^{4}$ & $8^{4}$ & $14^{4}$ & $16^{4}$ & $17^{4}$ \\
\hline
\end{tabular}

The superscripts of the question number indicate the original theoretical conception dimensions: ${ }^{1}$ cooperation \& support, ${ }^{2}$ active participation, ${ }^{3}$ parenting opposition, ${ }^{4}$ parenting conflict. 


\subsection{Confirmatory Factor Analysis}

Adopting Maximum Likelihood Estimation, we conducted confirmatory factor analysis on the theoretical assumption of exploratory factor analysis, in order to observe the rationality of the co-parenting structure. We respectively verified the large samples in Dalian and Benxi regions. Table 3 and Table 4 show the fitting situations of the study model. Then we found that the index of the initial model was relatively ideal and its degree of fitting was preferable. With no need of amending the model, we determined the co-parenting behavior questionnaire consisting of 20 questions.

The above two tables show that both of the verification models preferably explained the fitting relationship between the models and the data. The three indexes must be reported, CFI, IFI and NNFI, all exceed 0.9. Although RMSEA exceeds 0.05 , its upper limit doesn't exceed 0.08, so it's also acceptable. The three-dimensional model of the co-parenting behavior for primary school students and empirical data fit preferably, which indicates that the theoretical model we raised is rational and effective.

\subsection{Reliability of the Questionnaire}

The reliability of the questionnaire adopts homogeneity reliability, split-half reliability and test-retest reliability. The results of Table 5 show that the questionnaire has preferable reliability index in the overall passing dimension.

\subsection{Validity of the Questionnaire}

The validity evaluation of this study adopts four indexes: content validity, structure validity, congruent validity and construct validity.

\subsubsection{Content Validity}

The questions of the questionnaire of this study come from systemizing the results of literature review, openended questionnaire and interview survey. We invited the teachers and postgraduates in this field to deliberate, examine and modify the readability, representativeness and appropriateness of the formal questions we had compiled, so that the questions of the questionnaire could reflect the real situation of the co-parenting for the primary school students. This questionnaire is of higher content validity.

Table 3. Fitting index of confirmatory factor analysis model of the co-parenting behavior questionnaires from benxi samples $(\mathrm{n}=312)$.

\begin{tabular}{ccccccccc}
\hline Model & $\mathrm{X}^{2}$ & $\mathrm{DF}$ & $\mathrm{X}^{2} / \mathrm{DF}$ & RMSEA & SRMR & CFI & IFI & NNFI \\
\hline Study Model & 387.00 & 167 & 2.317 & 0.065 & 0.063 & 0.95 & 0.95 & 0.95 \\
\hline
\end{tabular}

Table 4. Fitting index of confirmatory factor analysis model of the co-parenting behavior questionnaires from dalian samples $(n=504)$.

\begin{tabular}{ccccccccc}
\hline Model & $\mathrm{X}^{2}$ & $\mathrm{DF}$ & $\mathrm{X}^{2} / \mathrm{DF}$ & RMSEA & SRMR & CFI & IFI & NNFI \\
\hline Study Model & 420.94 & 167 & 2.521 & 0.055 & 0.053 & 0.95 & 0.95 & 0.95 \\
\hline
\end{tabular}

Table 5. reliability coefficient of each subscale and total questionnaire of the co-parenting behavior questionnaire $(\mathrm{n}=828)$.

\begin{tabular}{cccc}
\hline & Alpha Coefficient & Split-half Reliability & Test-retest Reliability \\
\hline Cooperation \& Support & 0.7129 & 0.7199 & 0.701 \\
Initiative of Participation & 0.7880 & 0.7312 & 0.724 \\
Opposition \& Conflict & 0.8484 & 0.8004 & 0.780 \\
Total Questionnaire & 0.8775 & 0.8554 & 0.721 \\
\hline
\end{tabular}




\subsubsection{Structure Validity}

The results of confirmatory factor analysis indicates that all the indexes of the verification model consisting of 22 questions meet the requirement of statistics, and its fitting index is relatively preferable. It proves that this questionnaire is of preferable structure validity.

\subsubsection{Congruent Validity}

In the test, we also adopted the international universal authoritative test, i.e., the questionnaire of the Parenting Alliance (PAM), to investigate the congruent validity of the questionnaire of this study (Abidin \& Brunner, 1995). We calculated the Pearson correlation between the Parenting Alliance scale and the same self-compiled dimension of co-parenting behavior. The result showed that the population correlation of the same contents of the two questionnaires is 0.782 , and the correlation between the structures differed remarkably. It further proved the validity and rationality of the co-parenting behavior questionnaire.

\section{Discussions}

This study reveals that the co-parenting behavior structure consist of three aspects: cooperation and support, active participation, opposition and conflict. Seen from the family system theory, co-parenting mainly focuses on paying close attention to the tripartite interactions among the family subsystems.

The three co-parenting structure dimensions we reached have something consistent with the former studies, but there is also increase. For instance, in the former study on the three-dimensional structure, McHale (1995) considered that the three structural dimensions co-parenting behavior are consist of hostility-rivalry, domestic peace and the parents' parenting divergence (McHale, 1995). Margolin et al. (2001) raised three general dimensions of co-parenting in their studies: conflict, cooperation and triangle. The primarily imagined four dimensions in our study (cooperation and support, active participation, parenting opposition, divergence and conflicts) include these three aspects, i.e., cooperation and support is corresponding to domestic peace, parenting opposition is corresponding to hostility-rivalry, and divergence and conflict is corresponding to parenting divergence. Nothing but in the factor analysis process parenting opposition (hostility-rivalry) and parenting divergence are combined as one dimension. Our study takes in the dimension of active participation, because we consider that the co-parenting behavior itself has close connection with general parenting behaviors, and the parents' cooperation is actually the behavior needing their participation. Only participation itself can really reflect the tripartite interaction of the family system. The three-dimensional model of the co-parenting behavior structure discovered in this study is rational and effective.

Cooperation and support is the degree of mutual endeavor degree of cooperation and support in the tripartite interaction of parenting, which includes: undertaking one's own obligation in the aspects of child-minding, keeping house, guiding study and game activities and so on, as well as the couple' parenting objective and responsive and complementary degree towards the other one's request of assistance in the activity aspect. It mainly includes activity cooperation and emotional support, which are directly related to the practical co-parenting.

Active participation is the parents' manifestation and degree of participation in the aspect of exerting the family team effect, which mainly means the parents' participant initiative and positivity in the parent-child activities. It mainly includes the exertion of the leading function of parenting and whether the members of the family team can share the family parent-child activities, which is also closely relevant to the cooperation of co-parenting. What is different from cooperation and support is just that active participation emphasizes more of the investment of the parents' mental and physical efforts and the parents' participant initiative in the parent-child tripartite interaction.

Opposition and conflict is the inconformity of the parents' views on parenting questions and their parenting behaviors. It usually reveals the undesirable relationship in the tripartite interaction of the parents and the child. On one hand, it reflects that the parents misinterpret the limit with the child and attempt to ally the child to repel the other one, which is called as triangle in psychotherapy. One parent disturbs the other one's influence on the child, which mainly manifests as eliminating the other one's suggestion for the child or disturbing the child. He or she conducts active intervention in the direct activities of the other one with child. On the other hand, it manifests as behavioral rivalry or verbal quarrel.

The concrete manifestations include the couple's external rivalry, hostility, interruption or irony in the aspect of parenting strategic action. Parent often satirize, antagonize, talk about, criticize or belittle each other in the 
conversation. Mainly considered from the angle of family members' structure contradiction in the daily parenting, it includes the adverse expressions to the family solidarity, most of which is verbal expression, such as raking up the other one's faults and talking of the other one behind his or her back. Of course there is also expression of negative emotion, but this study questionnaire doesn't refer to emotional problems.

As the three basic elements of co-parenting behavior, the aspects of parents' cooperation and support, active participation, opposition and conflict organically unify in the individual's psychology and behavior. Cooperation and support is the basis of co-parenting, also the prerequisite of participant initiative, because the subject of co-parenting is cooperative quality. Participant initiative is mainly the family interactive mode, which is trilateral and imperceptible in the whole family interaction. It can manifest the couple's emotional attitude toward maintaining the family team. If cooperation and support, and active participation are the positive aspects of co-parenting, then opposition and conflict is the negative aspect of it, which is the reflection of undesirable parent-child relationship in the family triangle. But opposition and conflict doesn't mean noncooperation or nonparticipation. Usually, if the parents do better in the aspects of cooperation and support, and participant initiative, then there is lesser opposition and conflict; on the contrary, if there is more opposition and conflict, then the parents do relatively poor in the aspects of cooperation and support, and participant initiative. Just as the viewpoint of the Taiwanese scholar Guanghui Ye (2000).

All above, from the large sample questionnaire survey to the small sample family situation game observation and joint interview of husband and wife, from the amount of research to the qualitative discussion, we firstly observed by a family game, watch video perception impression as a generalization of the evaluation by the qualitative analysis to the amount of inspection.

\section{Conclusion}

1) The questionnaire about elementary school students' parents shared that parenting has good reliability and validity, and can be used as a common urban parenting assessment tool.

2) The co-parenting behavior is multidimensional integral. The three factors in the structure included collaboration support, active participation and conflict of opposites which are mutual independence and mutual relationship.

\section{References}

Abeer, A., \& Jerome, M. F. (2003). Factors Relevant to Parental Alliance for Parents of Children with Disabilities. Journal of Applied Rehabilitation Counseling, 34, 10.

Abidin, R. R., \& Brunner, J. F. (1995). Development of a Parenting Alliance Inventory. Journal of Clinical Child Psychology, 24, 31-40. http://dx.doi.org/10.1207/s15374424jccp2401_4

Brody, G. H., Stoneman, Z., Flor, D., McCrary, C., Hastings, L., \& Conyers, O. (1994). Financial Resources, Parent Psychological Functioning, Parent Co-Caregiving, and Early Adolescent Competence in Rural Two-Parent African-American Families. Child Development, 65, 590-605. http://dx.doi.org/10.2307/1131403

Feinberg, M. E., \& Kan, M. L. (2008). Establishing Family Foundations: Intervention Effects on Coparenting, Parent/Infant Well-Being, and Parent-Child Relations. Journal of Family Psychology, 22, 253-263. http://dx.doi.org/10.1037/0893-3200.22.2.253

Karreman, A., Tuijl, C. V., \& Marcel, A. G. (2008). Parenting, Co-Parenting, and Effortful Control in Preschoolers. Journal of Family Psychology, 22, 30-40. http://dx.doi.org/10.1037/0893-3200.22.1.30

Kolaka, A. M., \& Vernon-Feagans, L. (2008). Family-Level Coparenting Processes and Child Gender as Moderators of Family Stress and Toddler Adjustment. Infant and Child Development, 17, 617-638. http://dx.doi.org/10.1002/icd.577

Konold, T. R., \& Abidin, R. R. (2001). Parenting Alliance: A Multifactor Perspective. Assessment, 8, 47-65. http://dx.doi.org/10.1177/107319110100800105

Lindsey, E. W., Gale, Y., \& Cowell, M. (2005). Correlates of Coparenting during Infancy. Family Relations, 54, 346-360. http://dx.doi.org/10.1111/j.1741-3729.2005.00322.x

Margolin, G., Gordis, E. B., \& John, R. S. (2001). Coparenting: A Link between Marital Conflict and Parenting in TwoParent Families. Journal of Family Psychology, 15, 3-21. http://dx.doi.org/10.1037/0893-3200.15.1.3

McConnell, M. C., \& Kerig, P. K. (2002). Assessing Coparenting in Families of School-Age Children: Validation of the Coparenting and Family Rating System. Canadian Journal of Behavioral Science Ottawa, 34, 44-58.

http://dx.doi.org/10.1037/h0087154 
McHale, J. (1997). Overt and Covert Coparenting Processes in the Family. Family Process, 36, 183-201. http://dx.doi.org/10.1111/j.1545-5300.1997.00183.x

McHale, J. (2007). Is Seeing Believing? Expecting Parents' Outlooks Coparenting and Later Coparenting Solidarity. Infant Behavior \& Development, 30, 63-81. http://dx.doi.org/10.1016/j.infbeh.2006.11.007

McHale, J. P. (1995). Coparenting and Triadic Interaction during Infancy: The Roles of Marital Distress and Child Gender. Developmental Psychology, 31, 985-996. http://dx.doi.org/10.1037/0012-1649.31.6.985

McHale, J., Kuersten-Jogan, R., Lauretti, A., \& Rasmussen, J. (2000). Parental Reports of Coparenting and Observed Coparenting Behavior during the Toddler Period. Journal of Family Psychology, 14, 220-236. http://dx.doi.org/10.1037/0893-3200.14.2.220

Russell, A., \& Russell, G. C. (1994) Coparenting Early School-Age Children: An Examination of Mother-Father Interdependence within Families. Development Psychology, 30, 757-770. http://dx.doi.org/10.1037/0012-1649.30.5.757

Schoppe-Sullivan, S. J., Manglsdorf, S. C., Brown, G. L., \& Sokolowski, M. S. (2007). Goodness-of-Fit in Family Context: Infant Temperament, Marital Quality, and Early Coparenting Behavior. Infant Behavior \& Development, 30, 82-96. http://dx.doi.org/10.1016/j.infbeh.2006.11.008

Schoppe-Sullivan, S. J., Weldon, A. H., Cook, J. C., Davis, E. F., \& Buckley, C. K. (2009). Coparenting Behavior Moderates Longitudinal Relations between Effortful Control and Preschool Children's Externalizing Behavior. Journal of Child Psychology and Psychiatry, 50, 698-706. http://dx.doi.org/10.1111/j.1469-7610.2008.02009.x

Van Egeren, L. A., \& Hawkins, D. P. (2004). Coming to Terms with Coparenting \& Colon; Implications of Definition and Measurement. Journal of Adult Development, 11, 165-178. http://dx.doi.org/10.1023/B:JADE.0000035625.74672.0b 